\title{
Implementation of Particle Swarm Optimization Algorithm in Matlab Code for Hyperelastic Characterization
}

\author{
Talaka Dya1 ${ }^{*}$, Bale Baidi Blaise1, Gambo Betchewe1, Mohamadou Alidou² \\ ${ }^{1}$ Faculty of Science, University of Maroua, Maroua, Cameroon \\ ${ }^{2}$ Ecole Nationale Superieure Polytechnique, University of Maroua, Maroua, Cameroon \\ Email: *c.talakadya@gmail.com, balebaidi.blaise@yahoo.com, gambobetch@yahoo.fr, alidou.mohamadou fs.univ-maroua.cm
}

How to cite this paper: Dya, T., Blaise, B.B., Betchewe, G. and Alidou, M. (2021) Implementation of Particle Swarm Optimization Algorithm in Matlab Code for Hyperelastic Characterization. World Journal of Mechanics, 11, 146-163.

https://doi.org/10.4236/wjm.2021.117011

Received: April 13, 2021

Accepted: July 23, 2021

Published: July 26, 2021

Copyright () 2021 by author(s) and Scientific Research Publishing Inc. This work is licensed under the Creative Commons Attribution International License (CC BY 4.0).

http://creativecommons.org/licenses/by/4.0/

\begin{abstract}
The purpose of this paper is to demonstrate the applicability of Particle Swarm Optimization algorithm to determine material parameters in incompressible isotropic elastic strain-energy functions using combined tension and torsion loading. Simulation of rubber behavior was conducted from the governing equations of the deformation of a cylinder composed of isotropic hyperelastic incompressible materials. Four different forms of strain-energy function were considered based respectively on polynomial, exponential and logarithmic terms to reproduce load force $(\mathrm{N})$ and torque $(\mathrm{M})$ trends using natural rubber experimental data. After highlighting the minimization of the objective function generated in the fitting process, the study revealed that a particle swarm optimization algorithm could be successfully used to identify the best material parameters and characterize the behavior of rubber-like hyperelastic materials.
\end{abstract}

\section{Keywords}

Particle Swarm Optimization, Hyperelastic Models, Tension-Torsion Test, Load Force, Torsional Couple

\section{Introduction}

The study of the mechanical behavior of materials is of great importance to various industrial applications such as automation, aeronautics and biomechanics. The wide use of hyperelastic materials in industrial applications accounts for the high research interest expressed both in their characterization through experimental techniques and in the study of accurate constitutive models capable of 
describing their particular behaviour. Three criteria are necessary to determine the real behavior of a material: the choice of an adapted mechanical test, the choice of a constitutive law and the strategy of optimization of the material's parameters. The optimization of hyperelastic parameters is an essential step in the designing of industrial parts. Several numerical methods suitable for the collection of experimental data are available in the literature notably LevenbergMarquardt algorithm [1] [2] [3] [4], Beda-Chevalier method [5] [6] and the most recent methods such as Genetic Algorithm [7] [8] [9] (GA) and Particle Swarm Optimization method (PSO) [10] [11]. It has been shown that the PSO has better performance in finding the correct optimal solution than GA from a stochastic point of view [12]. The authors compared the performance of PSO and GA in terms of their accuracy, their robustness vis-à-vis population size and algorithm coefficients. Their study also revealed that not only does PSO have a higher probability to find the correct optimal solution than GA but is preferable to GA when time is a limiting factor. Without making a comparative study of optimization methods, this work seeks to demonstrate the applicability of the powerful PSO method to optimize the parameters of hyperelastic constitutive models.

The choice of an appropriate test is also essential to predict the mechanical behavior of hyperelastic materials. Materials' parameters were thus optimized using a combined tension-torsion test. Simultaneous tension-torsion experiments are a relevant alternative to consider multiple loading conditions [13] and guarantee the model's reliability to reproduce complex loading conditions. Several studies show that this type of test is well adapted to complex problems [14] [15] [16]. Lectez et al. [17] for example, use the tension-torsion test to characterize the behavior of elastometric engine mounts. Humphrey et al. [18] studied the finite extension and torsion of a nonlinear homogenous transversely isotropic incompressible solid cylinder. They proposed an analytical solution of the problem specifically for experimental correlation to identify a constitutive model for papillary muscles. These characterizations of rubbery materials on combined tension-torsional tests are reported in the literature. Emphasis is on the problem of torsion superimposed on the axial extension of a circular cylinder composed of an incompressible isotropic hyperelastic material [19]-[26].

This paper aims to implement PSO algorithm in Matlab code in order to determine the material parameters of the hyperelastic incompressible isotropic models using combined tension-torsion test data. The adopted method consists of reproducing trends of load force $(\mathrm{N})$ and torque $(\mathrm{M})$ of selected models using a solid circular cylinder's large deformations. To investigate PSO algorithm's appropriateness, the identification step of the material parameters is presented based on the correlation between the torsional couple (rather than the conventional methods of stress correlation or strain energy density correlation) rebuilt from test data and the theory. The identified parameters have been validated from the load force curves. Based on the combined tension-torsion test, the pre- 
dictions of the selected models concur with experimental data.

\section{Fundamentals}

Homogeneous large strain elastic materials such as elastomers and biological tissues are conveniently modeled by hyperelastic constitutive equations. Practically, there exists a strain energy density function $W$, such that the stress tensor its derivative with respect to the strain [27]. Let us denote $\boldsymbol{B}$ the left Cauchy-Green strain tensor defined as $\boldsymbol{F F}^{\mathrm{T}}, F$ being the deformation gradient tensor and ${ }^{\mathrm{T}}$ the transposition. Based on the assumption that elastomers are both isotropic and incompressible, this strain-energy function only depends on the two first invariants of the left $B$, i.e.

$$
W=W\left(I_{1}, I_{2}\right)
$$

with

$$
I_{1}=\operatorname{tr} B \quad \text { and } \quad I_{2}=I_{1}^{2}-\operatorname{tr}\left(\boldsymbol{B}^{2}\right) .
$$

The corresponding Cauchy stress tensor $\sigma$ is expressed as follows:

$$
\sigma=-p \boldsymbol{I}+2 \frac{\partial W}{\partial I_{1}} \boldsymbol{B}-2 \frac{\partial W}{\partial I_{2}} B^{-1},
$$

where $p$ is a hydrostatic pressure term associated with the incompressibility constraint and $\boldsymbol{I}$ is the identity tensor. The equations describing the tension-torsion deformation of an isochoric cylinder were derived by Rivlin and Saunders [19]. We consider a deformed circular cylinder of radius $R$, length $l$, subject to a combination of a tension load $N$ and a torsion torque $M$. The deformation consists in an axial displacement $u$ in the direction $e z$ (Figure 1). Let us assume that $(r, \theta, z)$ is the undeformed cylindrical coordinate and $(R, \Theta, Z)$, the coordinate in the deformed configuration. This can be written thus:

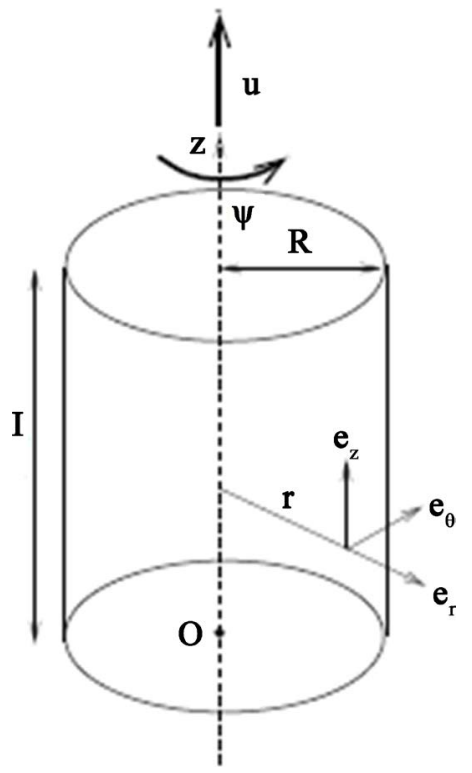

Figure 1. Circular cylinder subject to combined tension and torsion. 


$$
R=\frac{r}{\sqrt{\lambda}}, \quad \Theta=\theta+\Psi \lambda z \quad \text { and } \quad Z=\lambda z
$$

where the constant of proportionality $\Psi$ is termed the amount of torsion, $\theta$ the angle through which one end of the cylinder is rotated with respect to the other and $\lambda$ the global extension is given by

$$
\theta=\Psi l=\Psi \lambda L \text {. }
$$

Considering a cylinder subject to both extension and torsion, the deformation gradient tensor is:

$$
\boldsymbol{F}=\left[\begin{array}{ccc}
\lambda^{-1 / 2} & 0 & 0 \\
0 & \lambda^{-1 / 2} & \lambda^{1 / 2} \Psi r \\
0 & 0 & \lambda
\end{array}\right],
$$

and the left Cauchy-Green tensor $\boldsymbol{B}$ is:

$$
\boldsymbol{B}=\left[\begin{array}{ccc}
\lambda^{-1} & 0 & 0 \\
0 & \lambda^{-1}+\lambda \Psi^{2} r^{2} & \lambda^{3 / 2} \Psi r \\
0 & \lambda^{3 / 2} \Psi r & \lambda^{2}
\end{array}\right]
$$

Thus, the main strain invariants are reduced to

$$
I_{1}=\lambda \Psi^{2} r^{2}+\frac{2}{\lambda}+\lambda^{2} \quad \text { and } \quad I_{2}=\Psi^{2} r^{2}+\frac{1}{\lambda^{2}}+2 \lambda
$$

The resultant applied load force and torque necessary to maintain the deformation are given by:

$$
M=4 \pi \Psi \int_{0}^{a}\left(\frac{\partial W}{I_{1}}+\frac{1}{\lambda} \frac{\partial W}{I_{2}}\right) r^{3} \mathrm{~d} r
$$

and

$$
N=4 \pi\left(\lambda-\lambda^{-2}\right) \int_{0}^{a}\left(\frac{\partial W}{I_{1}}+\frac{1}{\lambda} \frac{\partial W}{I_{2}}\right) r \mathrm{~d} r-2 \pi \Psi^{2} \int_{0}^{a}\left(\frac{\partial W}{I_{1}}+\frac{2}{\lambda} \frac{\partial W}{I_{2}}\right) r^{3} \mathrm{~d} r
$$

\section{Hyperelastic Constitutive Models}

A broad collection of models are available in the literature. In this study four phenomenological models were used models:

Mooney-Rivlin model [28]

The mooney-rivlin model is rather simple and uses a reduced amount of parameters. It deals with moderate nonlinear and simple material behavior. Strainenergy density function based on two invariants of the right Cauchy-Green deformation tensor is defined as:

$$
W=C_{10}\left(I_{1}-3\right)+C_{01}\left(I_{2}-3\right),
$$

where $C_{10}$ and $C_{01}$ are the material parameters.

Yeoh model [29]

The Yeoh model is a reduced third-order polynomial based only on the first invariant of the right Cauchy-Green tensor. This model deals with high non-linear 
material behavior using only three parameters.

$$
W=C_{10}\left(I_{1}-3\right)+C_{20}\left(I_{1}-3\right)^{2}+C_{30}\left(I_{1}-3\right)^{3},
$$

where $C_{10}, C_{20}$, and $C_{30}$ are the material parameters.

\section{Gent and Thomas model [30]}

Considering the general form proposed by Rivlin and Saunders [17], Gent and Thomas proposed the following empirical strain energy function which involves only two material parameters and a logarithmic function of the second invariant:

$$
W=K_{1}\left(I_{1}-3\right)+K_{2} \log \frac{I_{2}}{3},
$$

where $K_{1}$ and $K_{2}$ are the material parameters.

Fung model [31]

Yuan-Cheng Fung, the Founder of Modern Biomechanics, formulated the famous exponential strain constitutive equation for preconditioned soft tissues. The expression of this strain energy density function is based only on the first invariant of the right Cauchy-Green tensor

$$
W=C\left(\exp ^{\beta\left(I_{1}-3\right)}-1\right),
$$

where $C$ and $\beta$ are the material constants.

\section{PSO Algorithm for Hyperelastic Characterization}

The ability of the different material models to reproduce any deformation curve depends on the definition of the values of the parameters associated to each model. Furthermore, the choice of these parameters depends on how a particular experiment is conducted and how the experimental data are processed [32] [33] [34] [35]. For an elastic material subject to large strains, the usual approach is to directly approximate the constants that appear in the mathematical model by employing numerical optimization techniques to minimize the residual between the stress-strain relation (for case of classical tests), the torsional couple or load force (for case of tension-torsion test, see for example [36] [37]) and the experimental data. Then, identification consists in relating the theoretical solution $M_{\text {model }}$ resulting from a model with the experimental curves represented by a couple of the point $\left(M_{\text {exp }}, \Psi_{\text {exp }}\right)$, with $i=1,2,3, \cdots, n$ the number of the experimental points. To quantify the gap between the two responses, the objective function $f_{o b j}$ is generally defined as:

$$
f_{\text {obj }}=\sum_{i=1}^{n}\left({ }_{\text {exp }}\left(\Psi_{i}\right)-{ }_{\text {model }}\left(\Psi_{i}\right)\right)^{2} .
$$

A PSO algorithm will be used to minimize the objective function and identify the material parameters to obtain a proper fitness of the curves. The load-force curves simulation response is fitted to validate the specified parameters by the PSO method employed.

The PSO algorithm was formulated by Edward and Kennedy in 1995 [38]. This algorithm is a stochastic optimization technique that is well adapted to the 
optimization of nonlinear functions in multidimensional space. It has been applied to several real world problems [39]. It is a class of swarm intelligence algorithms that attempts to reproduce the social behavior of a generic population. PSO population is called a swarm and each individual in the population of PSO is called a particle. All of the particles have fitness values evaluated by the fitness function to be optimized. They have velocities that direct the ?ying of the particles. The system is initialized with a population of random solutions and searches for optima by updating generations. To seek the optimal solution, each particle moves in the direction to its previously best ( Pbest) position and the global best (Gbest) position in the swarm. The speed and position of each particle change according to the following equality (see [40]):

$$
\begin{gathered}
V_{i, j}^{k+1}=w V_{i, j}^{k}+c_{1} r_{1}\left(\text { Pbest }_{i, j}^{k}-X_{i, j}^{k}\right)+c_{2} r_{2}\left(\text { Gbest }_{i, j}^{k}-X_{i, j}^{k}\right), \\
X_{i, j}^{k+1}=X_{i, j}^{k}+V_{i, j}^{k+1} .
\end{gathered}
$$

The index $i$ varies from 1 to $n$, whereas the index $j$ varies from 1 to $D . n$ is population size and $D$ is component of each individual of population. Pbest $t_{i, j}^{k}$ represents personal best $f^{\text {th }}$ component of $I^{\text {th }}$ individual, whereas Gbest $_{j}^{k}$ represents $f^{\text {th }}$ component of the best individual of population up to iteration $k$. $w$, the inertia weight used to balance the global exploration and local exploitation, $r_{1}$ and $r_{2}$ are uniformly distributed random variables within range [0,1], and $c_{1}$ and $c_{2}$ are positive constant parameters called acceleration coefficients.

\section{Application of the PSO to the Mooney-Rivlin model. \\ Set Parameters}

$F$ : objective fonction.

$n=100$ : population size.

$w_{\min }=0.4:$ minimal inertia weigh.

$w_{\max }=0.9:$ maximal inertia weigh.

$c_{1}=0.5:$ acceleration coefficient of particule.

$c_{2}=1$ : acceleration coefficient of essaim.

$\mathrm{Vit}_{\max }=1.5$ : maximal velocity.

$V i t_{\min }=-1.5:$ minimal velocity.

$I_{\max }=100:$ maximum number of iteration.

$C_{01 \min }=0$ : lower bound of variable $C_{01}$.

$C_{01 \max }=5:$ upper bound of variable $C_{01}$.

$C_{10 \min }=0$ : lower bound of variable $C_{10}$.

$C_{10 \max }=5$ : upper bound of variable $C_{10}$.

Initialize population of particles having positions and velocities

$$
\begin{aligned}
C_{01}= & C_{01 \min }+\left(C_{01 \max }-C_{01 \min }\right) * \operatorname{rand}(1, n) \\
& V C_{01}=V i t_{\text {min }} * C_{01} * \operatorname{rand}(1, n) \\
C_{10}= & C_{10 \min }+\left(C_{10 \max }-C_{10 \min }\right) * \operatorname{rand}(1, n) \\
& V C_{10}=V i t_{\min } * C_{01} * \operatorname{rand}(1, n)
\end{aligned}
$$




\section{Evaluation}

Set iteration $k=1$.

Calculate fitness of particles $F(C p)<F(C g)$; and find the index of the best particle $p=g$.

Update velocity and position of particles

$$
\begin{gathered}
V C_{01 i, j}^{k+1}=w * V C_{01 i, j}^{k}+C_{1} \text { rand }(1) *\left(C p_{01 b o n i, j}^{k}-C_{01 i, j}^{k}\right) \\
+C_{2} \text { rand }(1) *\left(C g_{01 b o n i, j}^{k}-C_{01 i, j}^{k}\right) \\
C_{01 i, j}^{k+1}=C_{01 i, j}^{k}+V C_{01 i, j}^{k+1} \\
V C_{10 i, j}^{k+1}=w * V C_{10 i, j}^{k}+C_{1} \operatorname{rand}(1) *\left(C p_{10 b o n i, j}^{k}-C_{10 i, j}^{k}\right) \\
+C_{2} \text { rand }(1) *\left(C g_{10 b o n i, j}^{k}-C_{10 i, j}^{k}\right) \\
C_{10 i, j}^{k+1}=C_{10 i, j}^{k}+V C_{10 i, j}^{k+1}
\end{gathered}
$$

with $w=w_{\text {min }}+\left(w_{\max }-w_{\min }\right) * i$ iter $/ I_{\max }$.

Evaluate fitness

$$
F\left(C p_{\text {bonend }}\right)=F\left(C g_{\text {bonend }}\right)
$$

If $k<I_{\max }$ then $k=k+1$ and goto step update velocity and position of particles else goto.

\section{Print optimal solutions}

$$
C p_{\text {bonend }}=C g_{\text {bonend }}
$$

\section{STOP}

The methodology presented in this section is summarized in the flowchart presented in Figure 2.

\section{Results and Discussion}

\subsection{Pure Torsion Application}

The application of combined finite axial stretch and finite torsion to a solid right cylinder of length 1 and radius is described in Rivlin's works (1949). The cylinder is first subjected to a simple extension in which its length increases and its radius decreases. Then, it is subjected to a simple torsion.

In the pure torsion case, the cylinder is maintained unextended, so that $\lambda=1$, Equations (9) and (10) become:

$$
M=4 \pi \Psi \int_{0}^{a}\left(\frac{\partial W}{I_{1}}+\frac{\partial W}{I_{2}}\right) r^{3} \mathrm{~d} r
$$

and

$$
-N=2 \pi \Psi^{2} \int_{0}^{a}\left(\frac{\partial W}{I_{1}}+2 \frac{\partial W}{I_{2}}\right) r^{3} \mathrm{~d} r
$$

The analytical expressions of the torsional couple $(M)$ and load force $(N)$ calculated for each model in this case are summarized in Table 1. 


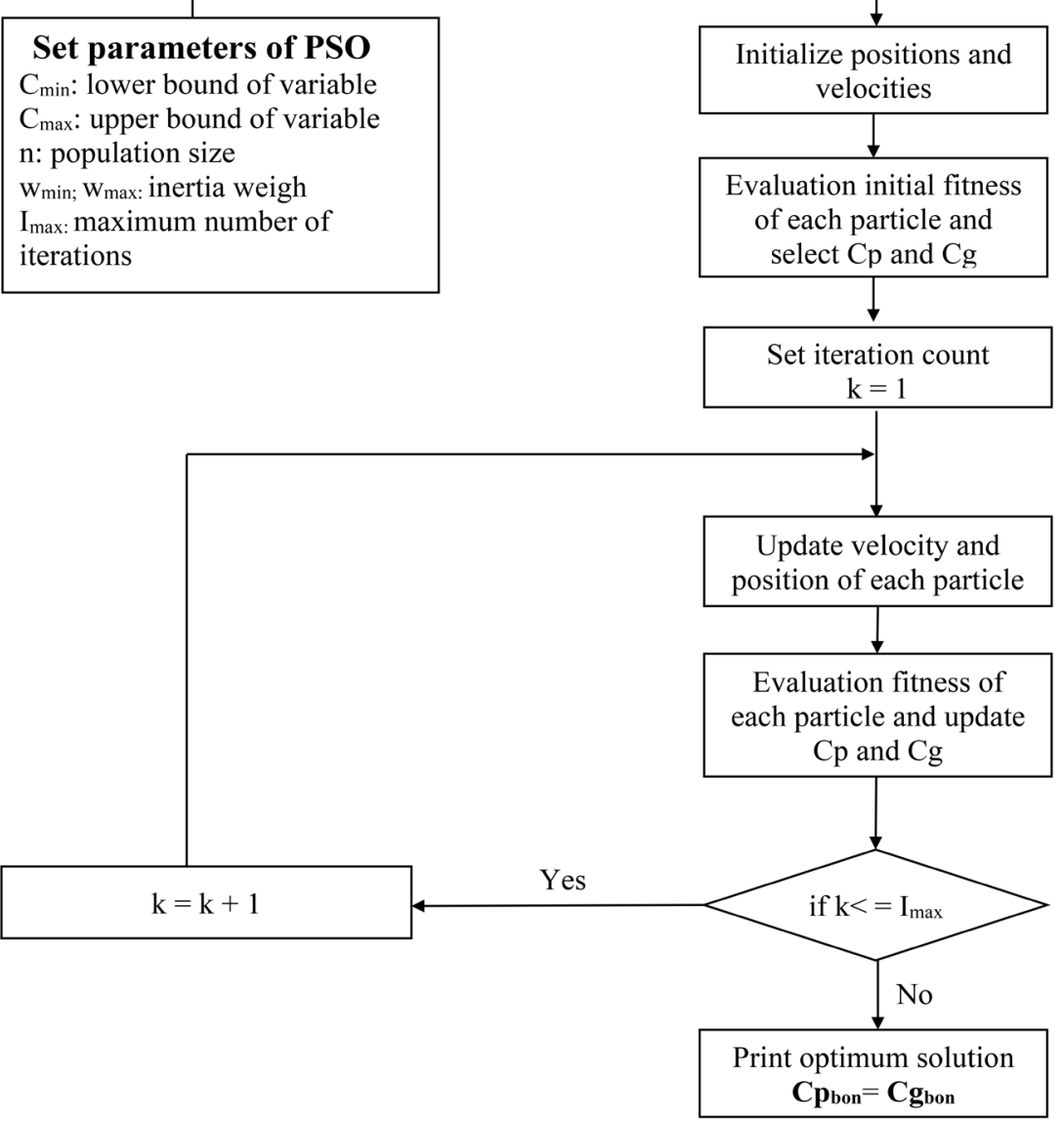

Figure 2. PSO Flowchart.

Table 1. Analytical solutions of load force $N$ and torque $M$ calculated on pure-torsion test.

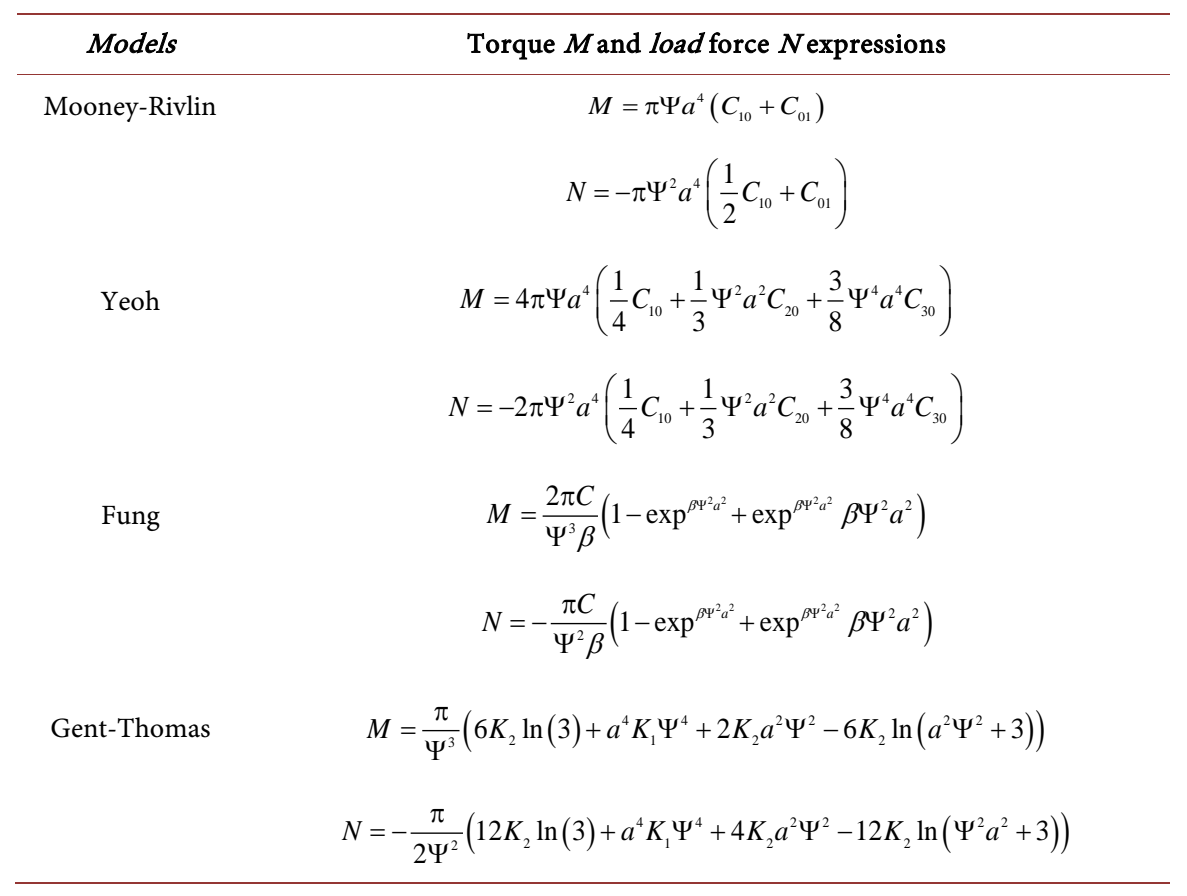




\subsubsection{Optimization of Material Parameters}

The optimization of the material parameters already described above is carried out in the case of pure torsion. The experimental data are those of Rivlin [41] and the analytical expressions of the torque are those given in Table 1. An optimization particle swarm algorithm was used to fit material constants' best values by minimizing the objective function.

\section{Fitness evaluation}

Finally, the algorithm must evaluate the fitness level between the experimental and theoretical curves. In this case, it is clear that the fitness function must be related to the error between the curve created with a set of optimized parameters and the data obtained with the real test. The process of fitness evaluation is aimed at determining the margin of error. So, when the error of the curve is near to zero, fitness is near to 100 .

Relative error is defined by

$$
\frac{|\Delta M|}{M}=\frac{\left|M_{\text {exp }}-M_{\text {model }}\right|}{M_{\text {exp }}}
$$

where $M_{\text {exp }}$ is the experimental torque and $M_{\text {model }}$, the theoretical predicted response of the model. The calculation of the relative errors generated in the fitting process presented in Figure 3 reveals that the relative errors are 0.012, 0.015, 0.016 and 0.016 for Mooney-Rivlin, Fung, Gent-Thomas and Yeoh models, respectively.

Both generated and approximated curves are presented in Figure 4 to show case the results obtained. From Figure 4, it can be observed that all the curves almost coincide along all the strain domain. It can thus be concluded that if

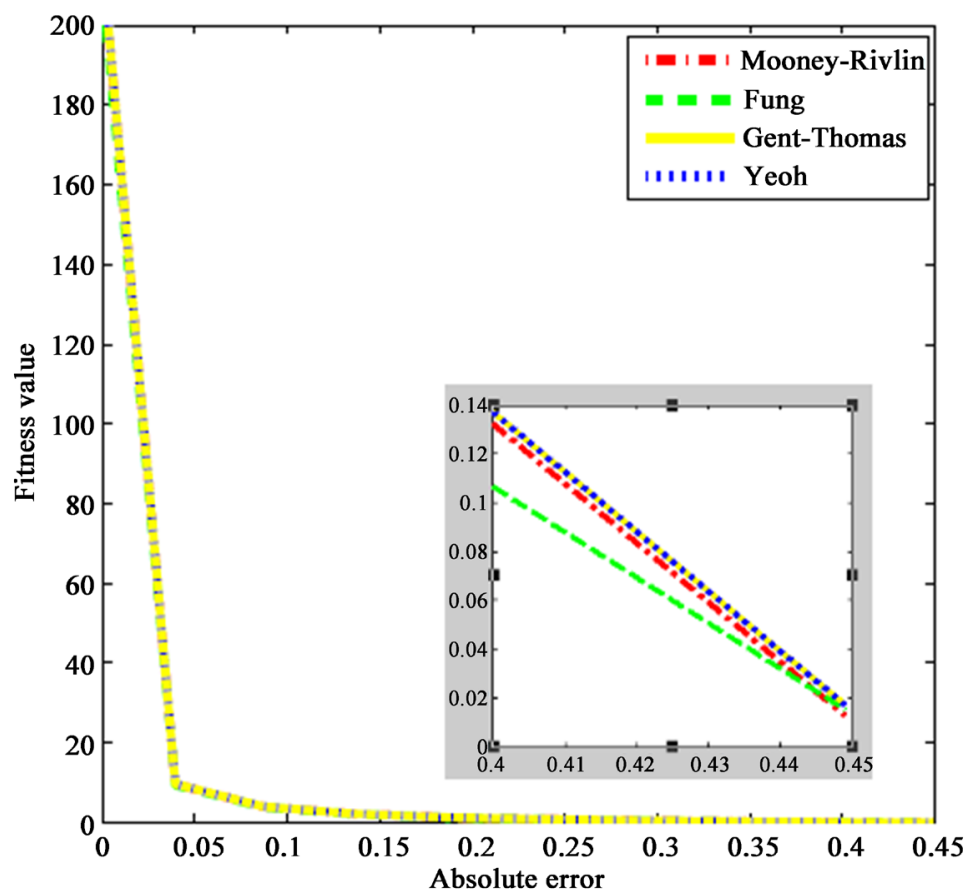

Figure 3. Plot of the relative error. 


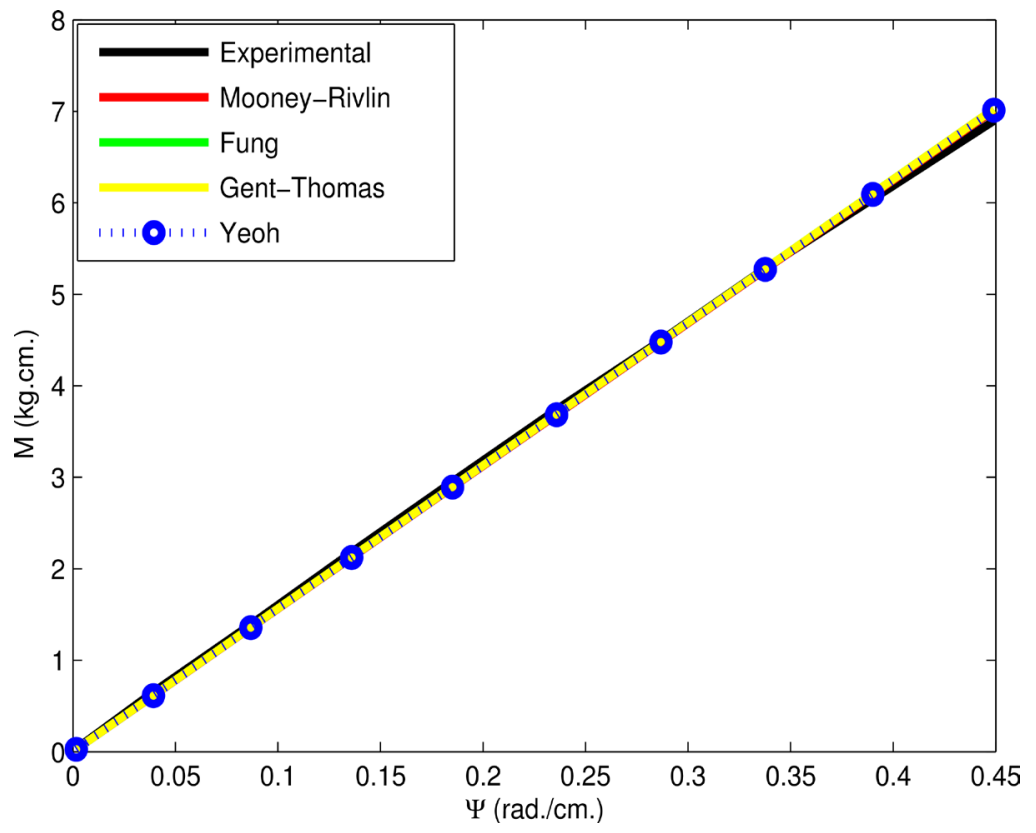

Figure 4. Best fit material parameters optimization: torsional torque curves on pure torsion test.

Table 2. Results of the identified parameters.

\begin{tabular}{cc}
\hline Models & Materials parameters optimized, $\mathrm{kPa}$ \\
\hline Mooney-Rivlin & $C_{10}=0.2914$ \\
& $C_{01}=0.09$ \\
\hline Yeoh & $C_{10}=0.38$ \\
& $C_{20}=1.06 \times 10^{-3}$ \\
& $C_{30}=4 \times 10^{-5}$ \\
\hline Fung & $C=283.038$ \\
& $\beta=0.1091$ \\
\hline Gent and Thomas & $K_{1}=0.2259$ \\
& $K_{2}=0.4990$ \\
\hline
\end{tabular}

enough amount of data is used to fit the curve, the results will converge and correctly match the input data. The quest in this work for a proper match between the experimental and theoretical torsional torque curve of each model, led to the determination of the values of the material parameters which are presented in Table 2.

\subsubsection{Verification of the Identified Material Parameters}

To verify the accuracy of the optimized parameters, the identified constants in Table 1 were employed to predict the corresponding responses of the load force curve $(\mathrm{N})$. The resulted calculated were compared with the data and plotted as presented in Figure 5. Based on the identified parameters, Figure 5 shows 
agreement between the experimental and calculated results curves. This suggests that the proposed identification method by using PSO is reliable and efficient.

\subsection{Characterization of Hyperelastic Models by Combined Tension-Torsion Test}

\section{Prediction of Tension-Torsion Behavior}

In the case of combined tension and torsion, the uniaxial extension is kept constant during torsion. The analytical expressions of the torque $\mathrm{M}$ of the deformed cylinder are presented with each model in Table 3 and the simulation results of

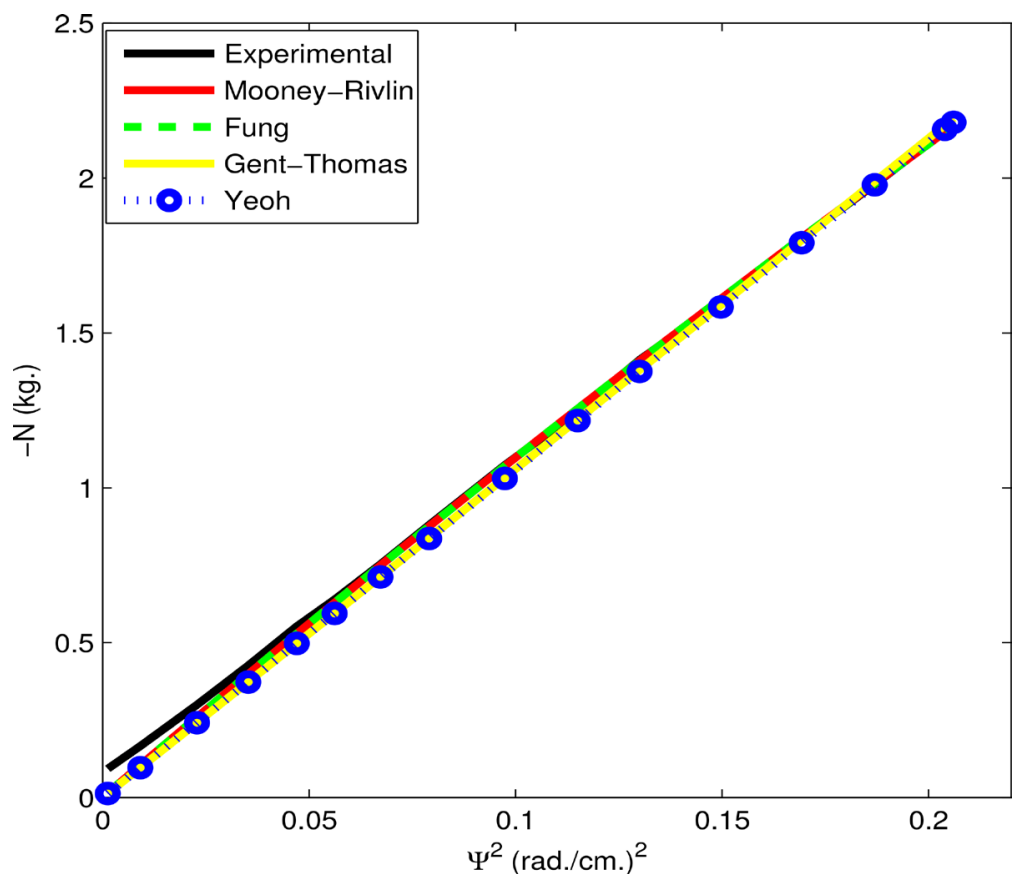

Figure 5. Verification of identified material parameters: load force curves on pure torsion test.

Table 3. Analytical solutions of torque $M$ calculated on combined tension and torsion loading.

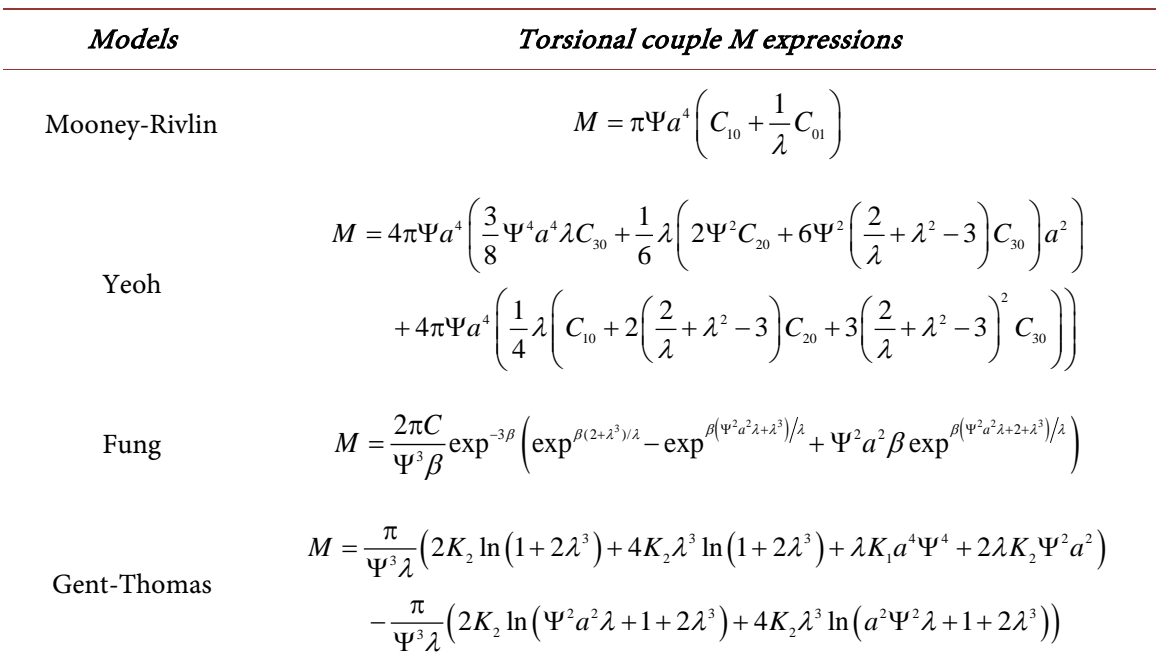


the torque in Figures 6-9.

To investigate the prediction power of the mechanical behaviour of hyperelastic models using combined tension and torsion test, the material parameters were determined for the first time by the PSO algorithm. This mechanical study shows that exponential and logarithmic strain energy functions do not give a good prediction of the tension-torsion test. The Fung (Figure 6) and Gent-Thomas (Figure 7)

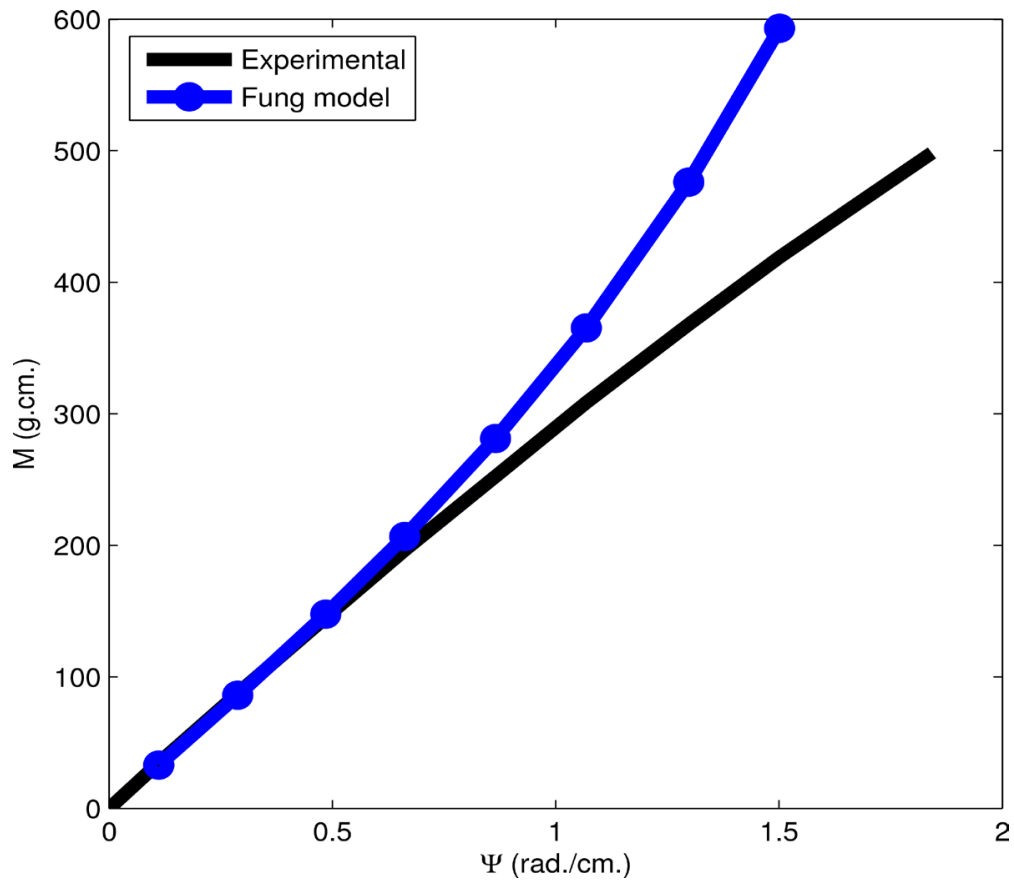

Figure 6. Prediction of tension-torsion curve by Fung model.

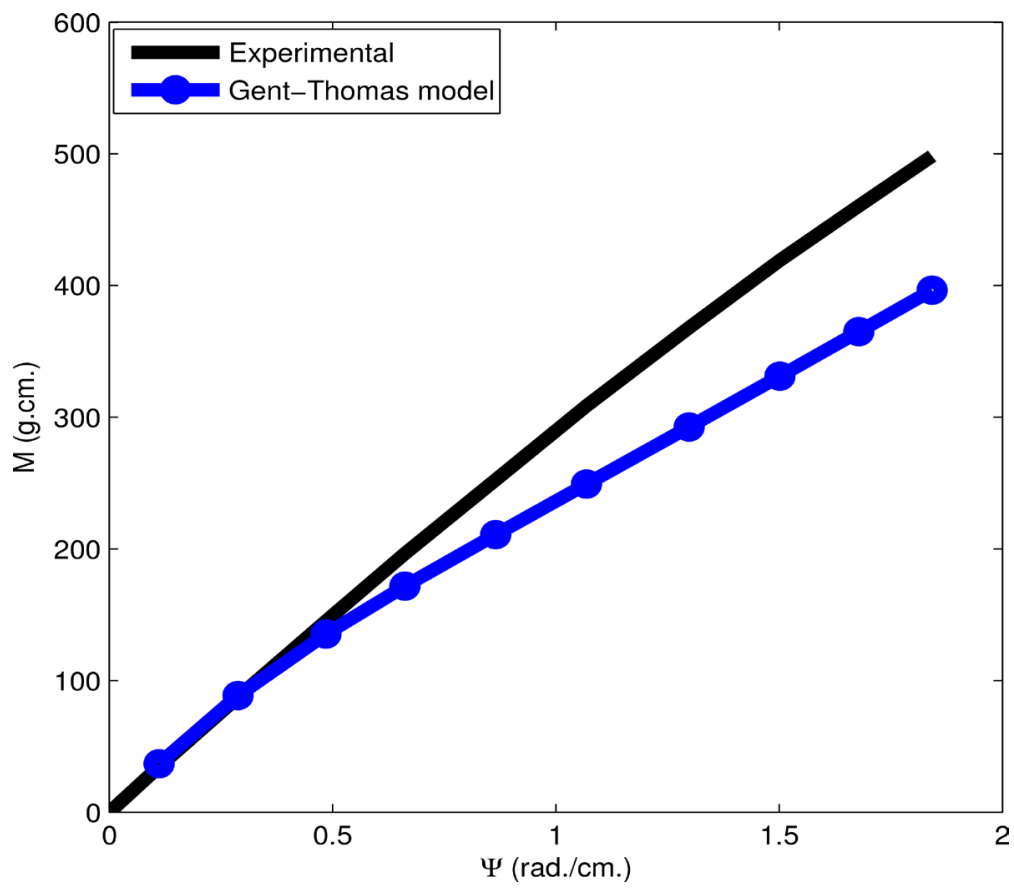

Figure 7. Prediction of tension-torsion curve by Gent-Thomas model. 


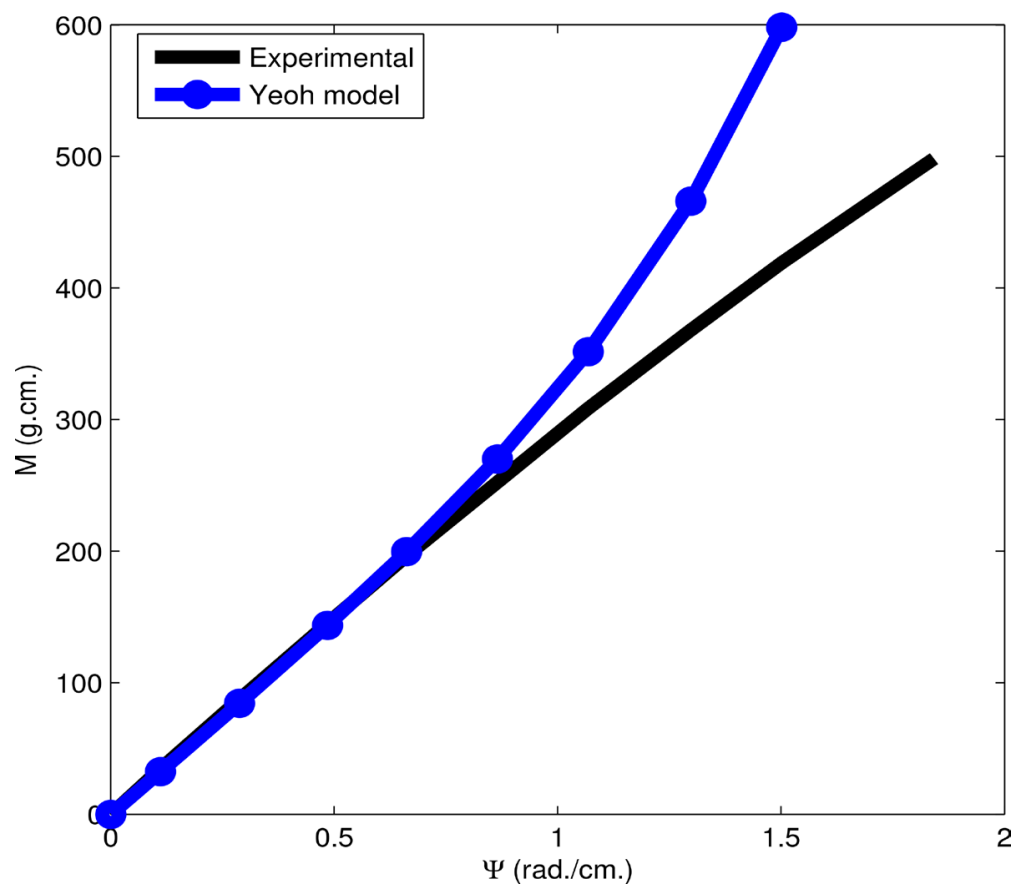

Figure 8. Prediction of tension-torsion curve by Yeoh model.

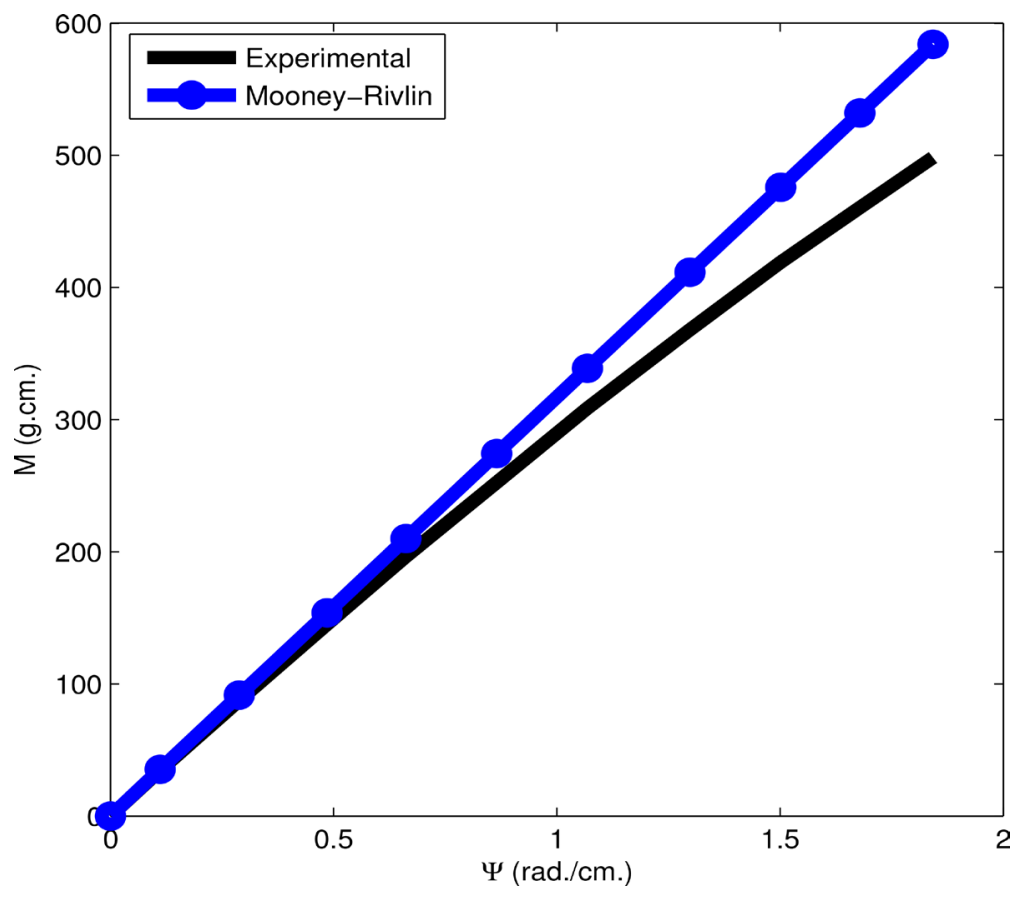

Figure 9. Prediction of tension-torsion curve by Mooney-Rivlin model.

models failed at small torsion angles. It is at intermediate angles that these two models reproduce the tensile-torsional test curve. The polynomial models are good models for the prediction of the tensile-torsional stress. Yeoh's model produces the tensile-torsional test up to a value of $\Psi=0.7 \mathrm{rad} / \mathrm{cm}$, then changes concavity (see Figure 8) due to the absence of $I_{2}$ in this model. Among the selected models, the Mooney-Rivlin model, the most accurate model to reproduce the shape 
of the experimental curve, as shown in Figure 9. The PSO method used in this work thus allowed for a qualitative mechanical characterization of the hyperelastic models.

\section{Conclusions}

The aim of this work was to identify the parameters of hyperelastic incompressible isotropic materials models using PSO algorithm through the combined tension and torsion loading test. Four classical hyperelastic models were used based on the simulation of their torque and force curves to determine that is the best theoretical model capable of reproducing the mechanical behavior for rubber-like materials under tension-torsion loading. An optimization particle swarm algorithm was used to fit material constants' best values on the pure torsion test. These parameters were then validated and used to simulate the tension-torsion behavior. All in all, the results of the study revealed that:

1) The combined tension and torsion test's mechanical behavior can be accurately adjusted by using the polynomial hyperelastic models with a good agreement with the Rivlin's data for the torsionnal couple curve;

2) The particle swarm optimization algorithm can be successfully used for hyperelastic characterization and for the generation of best material parameters;

3) The models capable of better reproducing the mechanical behavior of rubber materials from the combined tension and torsion test depend on the two invariants of Cauchy-Green $I_{1}$ and $I_{2}$.

\section{Conflicts of Interest}

The authors declare no conflicts of interest regarding the publication of this paper.

\section{References}

[1] Martins, P.A.L.S., Natal Jorge, R.M. and Ferreira, A.J.M. (2006) A Comparative Study of Several Material Models for Prediction of Hyperelastic Properties: Application to Silicone-Rubber and Soft Tissues. Strain, 42, 135-147. https://doi.org/10.1111/j.1475-1305.2006.00257.x

[2] Wu, Y., Wang, H. and Li, A. (2016) Parameter Identification Methods for Hyperelastic and Hyper-Viscoelastic Models. Applied Sciences, 6, 386. https://doi.org/10.3390/app6120386

[3] Nunes, L.C.S. (2011) Mechanical Characterization of Hyperelastic Polydimethylsiloxane by Simple Shear Test. Materials Science and Engineering, 528, 1799-1804. https://doi.org/10.1016/j.msea.2010.11.025

[4] Rachik, M., Schmidtt, F., Reuge, N., Le Maoult, Y. and Abbeé, F. (2001) Elastomer Biaxial Characterization Using Bubble Inflation Technique. II: Numerical Investigation of Some Constitutive Models. Polymer Engineering and Science, 41, 532-541. https://doi.org/10.1002/pen.10750

[5] Beda, T. (2005) Optimizing the Ogden Strain Energy Expression of Rubber Materials. Journal of Engineering Materials and Technology, 127, 351-353. https://doi.org/10.1115/1.1925282 
[6] Beda, T. (2006) Combining Approach in Stages with Least Squares for Fits of Data in Hyperelasticity. Comptes Rendus Mecanique, 334, 628-633. https://doi.org/10.1016/j.crme.2006.06.004

[7] Fernández, J.R., López-Campos, J.A., Segade, A. and Vilán, J.A. (2018) A Genetic Algorithm for the Characterization of Hyperelastic Materials. Applied Mathematics and Computation, 329, 239-250. https://doi.org/10.1016/j.amc.2018.02.008

[8] Blaise, B.B., Betchewe, G. and Beda, T. (2019) Optimization of the Model of Ogden Energy by the Genetic Algorithm Method. Applied Rheology, 29, 21-29. https://doi.org/10.1515/arh-2019-0003

[9] López-Campos, J.A., Segade, A., Casarejos, E., Fernández, J.R. and Días, G.R. (2019) Hyperelastic Characterization Oriented to Finite Element Applications Using Genetic Algorithms. Advances in Engineering Software, 133, 52-59. https://doi.org/10.1016/j.advengsoft.2019.04.001

[10] Alam, M.N. (2016) Particle Swarm Optimization: Algorithm and Its Codes in MATLAB.

[11] Ramzanpour, M., Hosseini-Farid, M., Ziejewski, M. and Karami, G. (2019) Particle Swarm Optimization Method for Hyperelastic Characterization of Soft Tissues. International Mechanical Engineering Congress and Exposition, Salt Lake City, 11-14 November 2019. https://doi.org/10.1115/IMECE2019-11829

[12] Duan, Y., Harley, R.G. and Habetler, T.G. (2009) Comparison of Particle Swarm Optimization and Genetic Algorithm in the Design of Permanent Magnet Motors. IEEE 6 th International Power Electronics and Motion Control Conference, Wuhan, 17-20 May 2009, 822-825. https://doi.org/10.1109/IPEMC.2009.5157497

[13] Rivlin, R.S. and Saunders, D.W. (1951) Large Elastic Deformations of Isotropic Materials VII. Experiments on the Deformation of Rubber. Philosophical Transactions of the Royal Society A, 243, 251-288. https://doi.org/10.1098/rsta.1951.0004

[14] Haupt, P. and Sedlan, K. (2001) Viscoplasticity of Elastomeric Materials. Experimental Facts and Constitutive Modeling. Archive of Applied Mechanics, 71, 89-109. https://doi.org/10.1007/s004190000102

[15] Mars, W.V. and Fatemi, A. (2004) A Novel Specimen for Investigating the Mechanical Behavior of Elastomers under Multiaxial Loading Conditions. Experimental Mechanics, 44, 136-146. https://doi.org/10.1007/BF02428173

[16] Suphadon, N., Thomas, A.G. and Busfield, J.J.C. (2009) Viscoelastic Behavior of Rubber under a Complex Loading. Polymer, 113, 693-699.

https://doi.org/10.1002/app.30102

[17] Lectez, A.S., Verron, E., Huneau, B., Béranger, A.S. and Le Brazidec, F. (2013) Characterization of Elastomers under Simultaneous Tension and Torsion for Application to Engine Mounts. In: Constitutive Models for Rubber VIII, CRC Press, Boca Raton, 585-590.

[18] Humphrey, J.D. (2002) Cardiovascular Solid Mechanics. Springer-Verlag, New York, 758-766. https://doi.org/10.1007/978-0-387-21576-1

[19] Rivlin, R.S. (1949) Large Elastic Deformations of Isotropic Materials VI. Further Results in the Theory of Torsion, Shear and Flexure. Philosophical Transactions of the Royal Society A, 242, 173-195. https://doi.org/10.1007/978-1-4612-2416-7_10

[20] Rivlin, R.S. and Saunders, D.W. (1951) Large Elastic Deformations of Isotropic Materials VII. Experiments on the Deformation of Rubber. Philosophical Transactions of the Royal Society A, 243, 251-288. https://doi.org/10.1007/978-1-4612-2416-7_12

[21] Sigaeva, T. and Czekanski, A. (2016) Coupling of Surface Effect and Hyperelasticity in Combined Tension and Torsion Deformations of a Circular Cylinder. Interna- 
tional Journal of Solids and Structures, 85-86, 172-179.

https://doi.org/10.1016/j.ijsolstr.2016.02.019

[22] Suphadon, N. and Busfield, J.J.C. (2009) Elastic Behaviour of Rubber Cylinders under Combined Torsion and Tension Loading. Plastics Rubber and Composites, 38 , 337. https://doi.org/10.1179/146580109X12473409436788

[23] Kashaev, R.M. (2008) On Tension-Torsion Testing of Solid Cylindrical Specimens. Letters on Materials, 8, 346-352.

https://doi.org/10.22226/2410-3535-2018-3-346-352

[24] Kirkinis, E. and Ogden, R.W. (2002) On Extension and Torsion of a Compressible Elastic Circular Cylinder. Mathematics and Mechanics of Solids, 7, 373-392.

https://doi.org/10.1177/108128028476

[25] Michelle, S., Fatt, H. and Ouyang, X. (2007) Integral-Based Constitutive Equation for Rubber at High Strain Rates. International Journal of Solids and Structures, 44, 6491-6506. https://doi.org/10.1016/j.ijsolstr.2007.02.038

[26] Lectez, A.S., Verron, E. and Huneau, B. (2014) How to Identify a Hyperelastic Constitutive Equation for Rubber-Like Materials with Multiaxial Tension-Torsion Experiments. International Journal of Non-Linear Mechanics, 65, 260-270. https://doi.org/10.1016/j.ijnonlinmec.2014.06.007

[27] Treloar, L.R.G., Hopkins, H.G., Rivlin, R.S. and Ball, J.M. (1976) The Mechanics of Rubber Elasticity [and Discussions]. Proceedings of the Royal Society A: Mathematical Physical and Engineering Sciences, 351, 301-330.

https://doi.org/10.1098/rspa.1976.0144

[28] Rivlin, R.S. (1948) Large Elastic Deformations of Isotropic Materials, I, II, III, Fundamental Concepts. Philosophical Transactions of the Royal Society A, 240, 459. https://doi.org/10.1098/rsta.1948.0002

[29] Yeoh, O.H. (1990) Characterization of Elastic Properties of Carbon-Black-Filled Rubber Vulcanizates. Rubber Chemistry and Technology, 63, 792-805. https://doi.org/10.5254/1.3538289

[30] Gent, A.N. and Thomas, A.G. (1958) Forms for the Stored (Strain) Energy Function for Vulcanized Rubber. Journal of Polymer Science, 28, 625-628. https://doi.org/10.1002/pol.1958.1202811814

[31] Fung, Y.C.B. (1967) Elasticity of Soft Tissues in Sample Elongation. American Journal of Physiology, 213, 1532-1544.

https://doi.org/10.1152/ajplegacy.1967.213.6.1532

[32] Chatelin, S., Constantinesco, A. and Willinger, R. (2010) Fifty Years of Brain Tissue Mechanical Testing: From in Vitro to in Vivo Investigations. Biorheology, 47, 255-276. https://doi.org/10.3233/BIR-2010-0576

[33] Evans, S.L. (2017) How Can We Measure the Mechanical Properties of Soft Tissues? In: Avril, S. and Evans, S.L., Eds., Material Parameter Identification and Inverse Problems in Soft Tissue Biomechanics, Springer, London, 67-83. https://doi.org/10.1007/978-3-319-45071-1_3

[34] Staber, B. and Guilleminot, J. (2017) Stochastic Hyperelastic Constitutive Laws and Identification Procedure for Soft Biological Tissues with Intrinsic Variability. Journal of the Mechanical Behavior of Biomedical Materials, 65, 743-752. https://doi.org/10.1016/j.jmbbm.2016.09.022

[35] Wex, C., Arndt, S., Stoll, A., Bruns, C. and Kupriyanova, Y. (2015) Isotropic Incompressible Hyperelastic Models for Modelling the Mechanical Behaviour of Biological Tissues: A Review. Biomedical Engineering/Biomedizinische Technik, 60, 577-592. https://doi.org/10.1515/bmt-2014-0146 
[36] Hartmann, S. (2001) Numerical Studies on the Identification of the Material Parameters of Rivlin's Hyperelasticity Using Tension-Torsion Tests. Acta Mechanica, 148, 129-155. https://doi.org/10.1007/BF01183674

[37] Lectez, A.S. (2014) Comportement multiaxial de pièces élastomères précontraintes: Application aux suspensions moteur. Mécanique des matériaux [physics.class-ph] Ecole Centrale de Nantes, 151-170.

[38] Kennedy, J. and Eberhart, R.C. (1995) Particle Swarm Optimization. Proceedings IEEE Conference on Neural Networks, Perth, 27 November-1 December 1995, 1942-1948.

[39] Boehner, K., DePaula, R., Dourish, P. and Sengers, P. (2007) How Emotion Is Made and Measured. International Journal of Human-Computer Studies, 65, 275-291. https://doi.org/10.1016/j.ijhcs.2006.11.016

[40] Shi, Y. and Eberhart, R. (1998) A Modified Particle Swarm Optimizer. IEEE International Conference on Evolutionary Computation, Anchorage, 4-9 May 1998, 69-73.

[41] Rivlin, R.S. (1948) Large Elastic Deformations of Isotropic Materials III. Some Simple Problems in Cylindrical Polar Coordinates. Philosophical Transactions of the Royal Society A, 240, 509-525. https://doi.org/10.1007/978-1-4612-2416-7_7 


\section{Appendix A. Matlab Code: Identification of Material Parameters by PSO Algorithm}

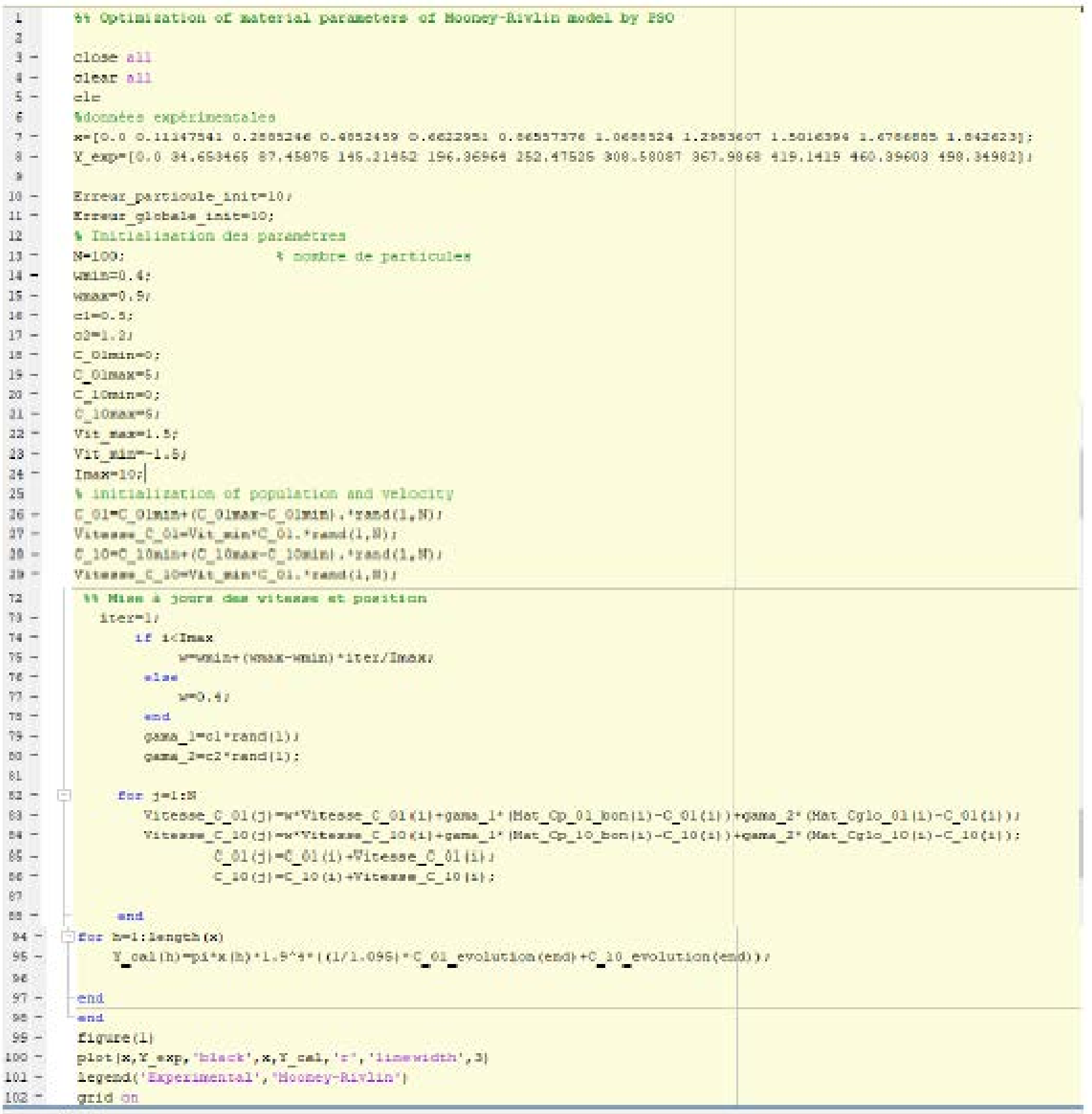

Commad Window

Erxeur_en_oours -

87.8462 